\title{
A GIANO-TNG high-resolution infrared spectrum of the airglow emission ${ }^{\star}$
}

\author{
E. Oliva ${ }^{1}$, L. Origlia ${ }^{2}$, R. Maiolino ${ }^{3}$, C. Baffa ${ }^{1}$, V. Biliotti ${ }^{1}$, P. Bruno ${ }^{4}$, G. Falcini ${ }^{1}$, V. Gavriousev ${ }^{1}$, F. Ghinassi ${ }^{5}$,

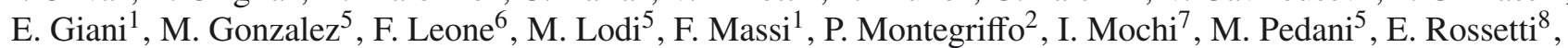 \\ S. Scuderi ${ }^{5}$, M. Sozzi ${ }^{1}$, A. Tozzi ${ }^{1}$, and E. Valenti ${ }^{9}$
}

\author{
1 INAF - Osservatorio Astrofisico di Arcetri, Largo E. Fermi 5, 50125 Firenze, Italy \\ e-mail: oliva@arcetri.inaf.it \\ 2 INAF - Osservatorio Astronomico di Bologna, via Ranzani 1, 40127 Bologna, Italy \\ 3 Cavendish Laboratory, University of Cambridge, 19 J. J. Thomson Avenue, Cambridge CB3 OHE, UK \\ ${ }^{4}$ INAF - Osservatorio Astrofisico di Catania, via S. Sofia 78, 95123 Catania, Italy \\ 5 INAF - Fundación Galileo Galilei, Rambla José Ana Fernández Pérez, 7, 38712 Breña Baja, TF, Spain \\ ${ }^{6}$ Dipartimento di Fisica e Astronomia, Sezione Astrofisica, Università di Catania, via S. Sofia 78, 95123 Catania, Italy \\ 7 Lawrence Berkeley National Laboratory, MS 2-400, One Cyclotron Road, Berkeley, CA 94720, USA \\ 8 Dipartimento di Astronomia, Università di Bologna, via Ranzani 1, 40127 Bologna, Italy \\ ${ }^{9}$ European Southern Observatory, Karl-Schwarzschild-Str. 2, 85748 Garching bei Muenchen, Germany
}

Received 26 February 2013 / Accepted 11 May 2013

\section{ABSTRACT}

\begin{abstract}
Aims. A flux-calibrated high-resolution spectrum of the airglow emission is a practical $\lambda$-calibration reference for astronomical spectral observations. It is also useful for constraining the molecular parameters of the $\mathrm{OH}$ molecule and the physical conditions in the upper mesosphere.

Methods. We used the data collected during the first technical commissioning of the GIANO spectrograph at the Telescopio Nazionale Galileo (TNG). The high-resolution $(R \simeq 50000)$ spectrum simultaneously covers the $0.95-2.4 \mu \mathrm{m}$ wavelength range. Relative flux calibration is achieved by the simultaneous observation of a spectrophotometric standard star.

Results. We derived a list of improved positions and intensities of $\mathrm{OH}$ infrared lines. The list includes $\Lambda$-split doublets, many of which are spectrally resolved. Compared with previous works, the new results correct errors in the wavelengths of the Q-branch transitions. The relative fluxes of $\mathrm{OH}$ lines from different vibrational bands show remarkable deviations from theoretical predictions: the $\Delta v=3$, 4 lines are a factor of 2 and 4 brighter than expected. We also found evidence of a significant fraction (1-4\%) of OH molecules with a non-thermal population of high- $J$ levels. Finally, we list wavelengths and fluxes of 153 lines not attributable to OH. Most of these can be associated with $\mathrm{O}_{2}$, while 37 lines in the $H$ band are not identified. The $\mathrm{O}_{2}$ and unidentified lines in the $H$ band account for $\simeq 5 \%$ of the total airglow flux in this band.
\end{abstract}

Key words. line: identification - infrared: general - techniques: spectroscopic

\section{Introduction}

The airglow emission is an annoying, unavoidable contamination of all ground-based astronomical observations. It mostly consists of narrow lines of molecular bands which, on the other hand, could be conveniently used as a reference spectrum to wavelength-calibrate spectroscopic data. The airglow lines at wavelengths $0.3-1.0 \mu \mathrm{m}$ were thoroughly compiled and modelled using high resolution $\left(R \simeq 10^{5}\right)$ data from HIRES-Keck and UVES-VLT; see e.g. Osterbrock et al. (1998), Hanuschik (2003), Cosby et al. (2006). The data in the near-infrared are much sparser and based on lower-resolution spectra, see e.g. Oliva \& Origlia (1992), Maihara et al. (1993). The most recent and complete line compilation is that of Rousselot et al. (2000, hereafter R2000), who used ISAAC-VLT spectra at resolving power $R \simeq 8000$. In addition to the relatively low resolution, which blends many lines, these spectra have a very limited simultaneous wavelength coverage $\Delta \lambda / \lambda=1 / 16$. Therefore, they

\footnotetext{
* Tables 1 and 3 are only available at the CDS via anonymous ftp to cdsarc.u-strasbg.fr (130.79.128.5) or via

http://cdsarc.u-strasbg.fr/viz-bin/qcat?J/A+A/555/A78
}

cannot be used to measure intensity ratios of a sufficiently large sample of lines, because the airglow intensity changes between the different exposures needed to cover the whole wavelength range. The ideal instrument for this type of measurement is a cross-dispersed spectrograph, which can combine high spectral resolution and broad wavelength coverage. GIANO-TNG is the first instrument of this type available to the astronomical community.

We present and discuss measurements taken with GIANO during its first technical commissioning at Telescopio Nazionale Galileo (TNG). In Sect. 2 we briefly describe the instrument, the measurements, and the data reduction. In Sect. 3 we present and discuss the results.

\section{Observations}

GIANO is a cross-dispersed spectrograph that produces in a single exposure a spectrum extending from $0.95 \mu \mathrm{m}$ to $2.4 \mu \mathrm{m}$ at a resolving power $R \simeq 50000$. The main disperser is a commercial R2 echelle grating with 23.2 lines $/ \mathrm{mm}$ that works in quasiLittrow configuration on a $d=100 \mathrm{~mm}$ collimated beam. Cross 


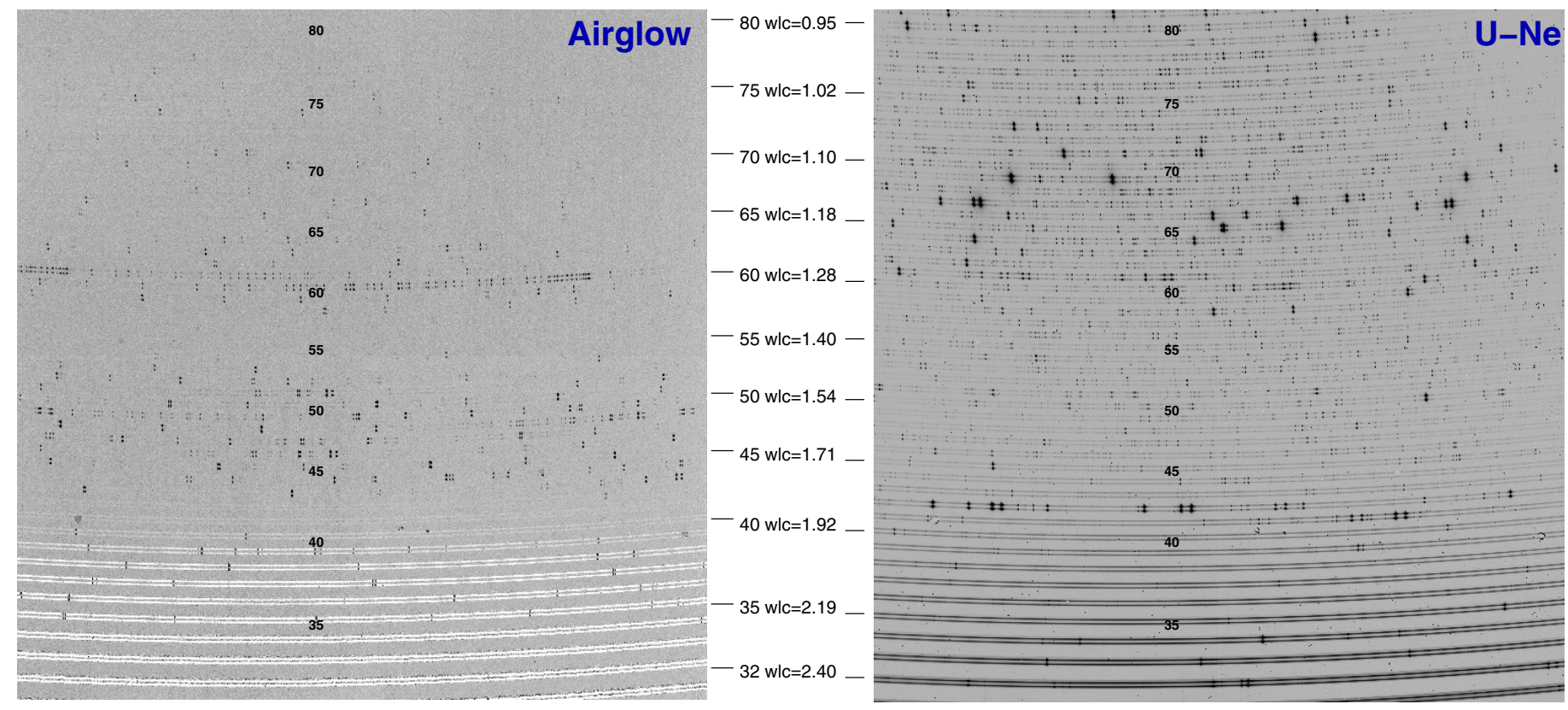

Fig. 1. GIANO echelle spectra of the airglow emission (left panel) and of the U-Ne calibration lamp (right-hand panel). The positions of several orders and the corresponding central wavelengths (in $\mu \mathrm{m}$ ) are marked.

dispersion is achieved via a network of fused silica and $\mathrm{ZnSe}$ prisms that work in double pass, i.e. they cross-disperse the light both before and after it is dispersed by the echelle gratings. This setup produces a curvature of the images of the spectral orders. More technical details on the instrument can be found in Oliva et al. $(2012 \mathrm{a}, \mathrm{b})$ and references therein.

The spectral layout on the detector is shown in Fig. 1. The echellogram on the detector spans 49 orders, from \#32 to \#80. The spectral coverage is complete up to $1.7 \mu \mathrm{m}$. At longer wavelengths the orders become wider than the detector. The effective spectral coverage in the $K$-band is about $75 \%$. The sky spectrum in the left panel of Fig. 1 was dark-subtracted using an exposure taken with a blocking filter at room temperature, for this reason, the thermal continuum beyond $2 \mu \mathrm{m}$ results in absorption.

For its first technical commissioning at the TNG, we used a bundle of two IR-transmitting ZBLAN fibres provisionally connected to the TNG focus for visiting instruments. These fibres are standard off-the-shelf products with a core of $85 \mu \mathrm{m}$, which corresponds to a sky-projected angle of 1 arcsec. The two fibres are aligned and mounted inside a custom connector. The cores are at a distance of $0.25 \mathrm{~mm}$, equivalent to a sky-projected angle of about 3 arcsec. Due to the constraints set by the visitor focus, the fibre entrances were coupled to the TNG using a provisional, simplified focal adapter that consisted of a commercial $\mathrm{CaF}_{2}$ singlet lens positioned $26 \mathrm{~mm}$ before the fibres. The focal adapter was mechanically mounted at a fiducial position, no other adjustment of the optical axis was possible. Unfortunately, this resulted in a very reduced efficiency of the system, which severely limited the use of the instrument for observations of faint targets. A pellicle beam-splitter, positioned just before the lens, was used to deviate $\sim 8 \%$ of the light to the guider CCD camera, working in the $Z$-band. Light from calibration lamps could be fed into the fibres by inserting a mirror in place of the beam-splitter.

The data were collected during part of the technical nights from July 27 to July 30, 2012. Sky-only spectra, such as those shown in Fig. 1, were collected by pointing at blank sky positions. Sky+star spectra were collected by centring a hot star with known flux (Hip 89584) in one of the two fibres. These spectra were used to measure the relative fluxes of the airglow lines. The flux calibration did not include correction for telluric absorption features, because the lines are not resolved. The geometry of the orders was determined using flat exposures with a tungsten calibration lamp. The $2 \mathrm{D}$ spectrum was thus rectified and the spectra were extracted by summing 6 pixels around each fibre, in the direction perpendicular to dispersion. Wavelength calibration was determined feeding the fibres with the light from a U-Ne lamp. The wavelengths of the uranium lines were taken from Redman et al. (2011), while for neon we used the table available on the NIST (Kramida et al. 2012). The $\lambda$ vs. pixel relationship was obtained starting from a physical model of the instrument. This procedure is part of the pipeline that we are developing for the instrument. The resulting wavelength accuracy was about $\lambda / 300000 \mathrm{rms}$, i.e. $0.05 \AA$ for lines in the $H$-band.

Relative flux calibration was performed by approximating the photon flux of the standard star (Hip 89584) with the following interpolation formula:

$\log \left(N_{\lambda}\right)=-3.46-1.58 \log \lambda-1.93(\log \lambda)^{2}$,

where $\lambda$ is in $\mu \mathrm{m}$ and $N_{\lambda}$ is in photons $/ \mathrm{cm}^{2} / \mathrm{s} / \mu \mathrm{m}$. The accuracy of the measured flux of bright lines in regions free of telluric absorptions is $10 \% \mathrm{rms}$.

\section{Results}

A total of about 750 airglow lines were detected in our spectra. About 500 can be attributed to $\mathrm{OH}$ roto-vibrational transitions, 114 can be associated with $\mathrm{O}_{2}$, while the others are unidentified.

We first concentrate on the $\mathrm{OH}$ lines, for which a rich theoretical background exists in the literature.

\section{1. $\mathrm{OH}$ wavelengths and fluxes}

Table 1 includes the lines that were unambiguously identified as $\mathrm{OH}$ transitions. For each $\Lambda$-doublet of $\mathrm{OH}$ lines we give the 
E. Oliva et al.: A GIANO-TNG high-resolution infrared spectrum of the airglow emission

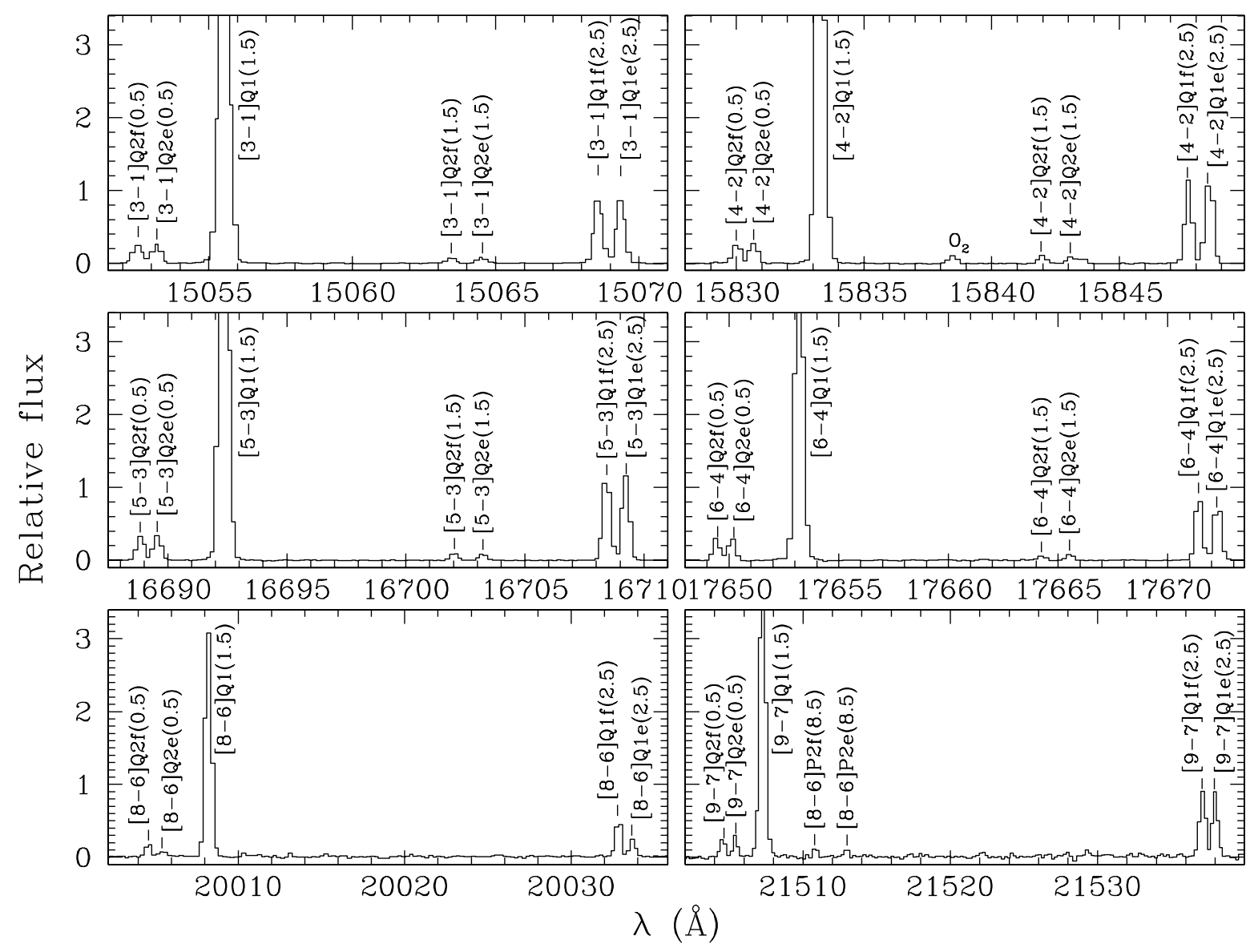

Fig. 2. Parts of the extracted spectrum including the Q-branch lines. Most of the $\Lambda$-doublets are clearly resolved at the GIANO resolution.

wavelengths (in vacuum) and the total flux of the doublet. The relative intensities of the two $e, f$ lines of each doublet, when resolved, were in all cases found to be equal to unity, within the errors.

The listed wavelengths are derived from the $\mathrm{OH}$ energy level positions of Abrams et al. (1994). These are the most accurate molecular data available in the literature and yield line wavelengths with an rms accuracy of $0.0035 \mathrm{~cm}^{-1}$, equivalent to $0.01 \AA$ at $16000 \AA$. Compared with the work of R2000, we find a discrepancy in the positions of all Q-lines. Specifically, we confirm that the $\Lambda$-doublets of most of these lines are clearly resolved at the GIANO resolution (see Fig. 2). In contrast to our results, these lines were predicted to be unresolved by the R2000 list. Similar discrepancies for a few Q lines were also reported by Ellis et al. (2012).

The intensities of the lines are expressed in units of photons $/ \mathrm{cm}^{2} / \mathrm{s}$, normalised to the intensity of the brightest line, which is set to $10^{3}$. The superscripts to the line intensities are used to flag the reliability of the flux measurement. Their meanings are as follows:

$a$ : well-detected line in a region free of telluric absorption. The line is free from blending or can be de-blended. The error on its relative flux is expected to be within $10 \% \mathrm{rms}$.

$b$ : well-detected line, but affected by some telluric absorption and/or blending and/or other problems. The error on its relative flux could be much larger than $10 \%$.

$c$ : line flux poorly defined because the line is detected at low signal-to-noise ratio, is severely affected by telluric absorption, or is strongly blended.

\subsection{Excitation and physical conditions of $\mathrm{OH}$}

The physical conditions of the $\mathrm{OH}$ molecules can be determined by computing the relative populations of the upper levels of the transitions, and comparing them with thermal distributions. For this computation we used the most up-to-date values of transition probabilities, i.e. those of van der Loo \& Groenenboom (2007). The results are shown in Fig. 3 along with theoretical curves (solid lines) for a thermalised population with a vibrational temperature $T_{\mathrm{vib}}=9000 \mathrm{~K}$ and a much lower rotational temperature $T_{\text {rot }}=180 \mathrm{~K}$, i.e. for typical excitation conditions (see e.g. R2000). The most striking result is the flattening towards higher energies in the observed distributions. This strong deviation from a thermal distribution is disclosed by the measurement of lines arising from levels with a rotational quantum number as high as $J=15.5$. Some of these lines are visible in the top panel of Fig. 3 and in the central panel of Fig. 6.

In Fig. 3 we also plot as dashed lines the level population expected, adding a certain fraction of hot molecules with $T_{\text {rot }}=T_{\text {vib. }}$. The results of this simple model fit the observations remarkably well, but it requires that the fraction of hot molecules increases with increasingly lower vibrational states. The results can be explained as follows. In the upper part of the mesosphere, the $\mathrm{OH}$ molecule is primarily formed by the reaction

$\mathrm{O}_{3}+\mathrm{H} \rightarrow \mathrm{OH}^{*}(v \leq 9)+\mathrm{O}_{2}$.

The freshly formed $\mathrm{OH}^{*}$ molecule is in a excited vibrational and rotational state. At the typical densities of the mesosphere, collisional de-excitations within a given vibrational state are much 


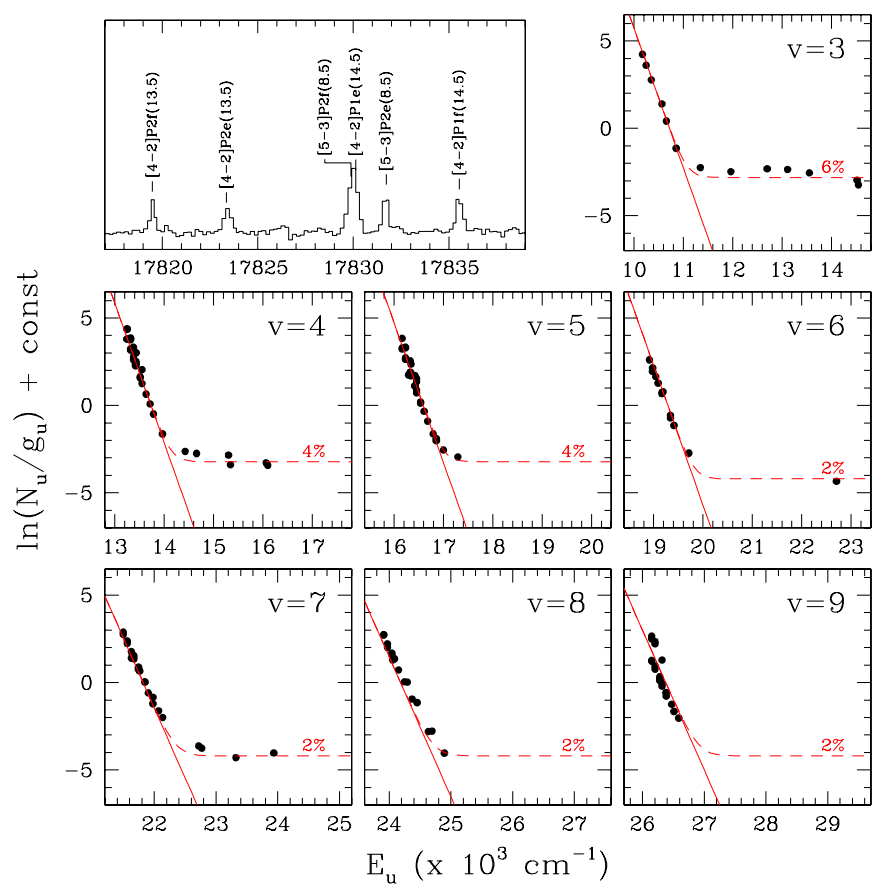

Fig. 3. Relative populations of the $\mathrm{OH}$ levels. $E_{\mathrm{u}}$ is the energy of the upper level (units of $10^{3} \mathrm{~cm}^{-1}$, equivalent to $1.99 \times 10^{-13} \mathrm{erg}$ ) and $g_{\mathrm{u}}$ is its statical weight. The solid lines are the values expected for a thermal population with a vibrational temperature $T_{\text {vib }}=9000 \mathrm{~K}$ and a rotational temperature $T_{\text {rot }}=180 \mathrm{~K}$. The dashed lines include a given fraction of hot molecules with $T_{\mathrm{rot}}=T_{\mathrm{vib}}$. The upper-left panel shows a region of the spectrum with some of the high-excitation lines from non-thermal levels.

faster than spontaneous transitions (see e.g. Sharma 1985). This process thermalises the rotational levels of most $\mathrm{OH}$ molecules to the gas temperature. The non-thermal lines that we detect come from the small fraction of $\mathrm{OH}^{*}$ molecules that spontaneously decay before thermalising. The fact that this fraction increases for lower vibrational states may indicate that the efficiency of collisional de-excitations decreases for lower $v$.

There are a few points at low energies in the $v=9$ subplot of Fig. 3 that appear to be offset from the solid red line. These are the $\Delta v=4$ transitions, which we discuss in the next section.

\subsection{Comparison with computed $\mathrm{OH}$ transition probabilities}

Another intriguing result follows from the analysis of the line fluxes arising in transitions from the same upper level. An excited molecule with a vibrational quantum number $v^{\prime}$ and rotational quantum number $J^{\prime}$ can spontaneously decay to a lower vibrational state $v^{\prime \prime}=v^{\prime}-\Delta v$ with rotational quantum numbers $J^{\prime \prime}=J^{\prime}-1$ (R line), $J^{\prime \prime}=J^{\prime}(\mathrm{Q}$ line $)$, and $J^{\prime \prime}=J^{\prime}+1$ (P line). Therefore, depending on the value $J^{\prime}$, there are two $(\mathrm{P}+\mathrm{Q})$ or three $(\mathrm{P}+\mathrm{Q}+\mathrm{R})$ lines for each $\Delta v$ band (for a complete scheme of the $\mathrm{OH}$ transition network see Fig. 2 of R2000). Since these lines are optically thin, their photon fluxes are simply determined by the population of the upper level $N_{\mathrm{u}}$ times the transition probability $A_{\mathrm{ul}}$, i.e.

$I=N_{\mathrm{u}} A_{\mathrm{ul}}$.

A convenient method for comparing observations with theoretical computations is to plot the value of $N_{\mathrm{u}}$ derived from different lines sharing the same upper level. The results are shown in Fig. 4. For each excited state, identified by its energy $E_{\mathrm{u}}$, we included all lines with reliable flux measurements.

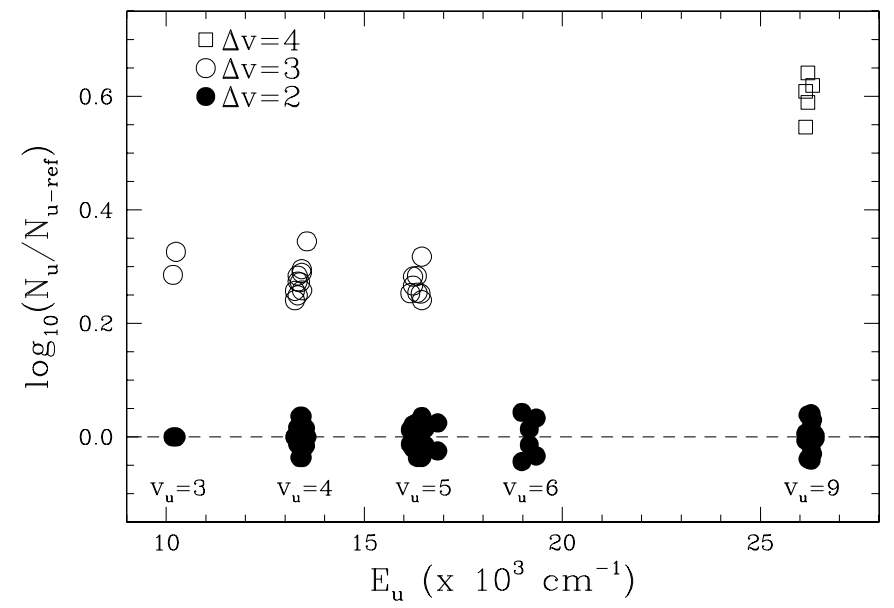

Fig. 4. Comparison between observed and predicted flux ratios of $\mathrm{OH}$ lines from the same upper levels. Points with $N_{\mathrm{u}} / N_{\mathrm{u}-\mathrm{ref}}$ far from unity (dashed line) indicate a discrepancy between observations and theoretical computations.

The computed value of $N_{\mathrm{u}}$ was normalised to the value derived from the brightest line i.e.

$Y=\log \left(N_{\mathrm{u}} / N_{\mathrm{u}-\mathrm{ref}}\right)_{i}=\log \left(I / A_{\mathrm{ul}}\right)_{i}-\log \left(I / A_{\mathrm{ul}}\right)_{\mathrm{ref}}$,

where the suffix $i$ refers to the line under consideration. Values of $Y$ close to zero imply good agreement between observations and theory. This is the case for all points relative to the $\Delta v=2$ lines (filled dots in Fig. 4), which are distributed around the $Y=0$ line with a scatter compatible with the observational errors.

The unexpected result is the systematic displacement of the other points. The $\Delta v=3$ lines are clustered around $Y=0.3$, while the $\Delta v=4$ transitions have an average value of $Y=0.6$. In either case the scatter of the points around their average values is compatible with the observational errors. This result indicates that the computed transition probabilities of the $\Delta v=3$ and $\Delta v=4$ lines are systematically underestimated by a factor of about 2 and 4, respectively. Very similar results are found using the most recent transition probabilities, published by van der Loo \& Groenenboom (2007), and the older values, published by Mies (1974). The strong discrepancy of lines with different $\Delta v$ is also evident in Table 2, which lists the observed and predicted ratios for selected pairs of lines from the same upper level.

We were indeed very surprised by this result, to the point of questioning the flux calibration of our data. In addition to double-checking the entire data reduction, we searched for other independent data that could provide precise quantitative information on the relative fluxes of $\Delta v=2,3,4$ bands of $\mathrm{OH}$.

We used archive TNG data taken with the Amici disperser of NICS, the TNG Near Infrared Camera Spectrometer (Baffa et al. 2001). These spectra simultaneously cover the $0.9-2.5 \mu \mathrm{m}$ wavelength range at a resolving power $R \simeq 50$. Although the resolution is by far too low to measure the intensities of the single $\mathrm{OH}$ lines, the spectra can be conveniently used to derive the integrated intensities and colours of the airglow within the infrared photometric bands. The results for a typical dark night are displayed in Fig. 5. The emission in the $Y(0.97-1.07 \mu \mathrm{m})$, $J(1.17-1.33 \mu \mathrm{m})$, and $H(1.48-1.78 \mu \mathrm{m})$ photometric bands is dominated by airglow lines, while $K^{\prime}(1.95-2.30 \mu \mathrm{m})$ also includes thermal emission from the telescope mirrors. While the temporal variation in the airglow-dominated bands is quite significant (up to $1 \mathrm{mag}$ ), the colours are much more stable, and can be conveniently used to compare with theoretical predictions. The $J-H$ colour is difficult to model because of the 
Table 2. Photon flux ratios of $\mathrm{OH}$ lines from the same upper level.

\begin{tabular}{lccc}
\hline \hline Line ratio $^{1}$ & Observed & \multicolumn{2}{c}{ Predicted $^{2}$} \\
\hline [3-0]P1(2.5)/[3-1]Q1(1.5) & 0.045 & 0.024 & 0.017 \\
[3-0]P1(3.5)/[3-1]Q1(2.5) & 0.15 & 0.071 & 0.051 \\
[4-1]Q1(1.5)/[4-2]Q1(1.5) & 0.11 & 0.063 & 0.060 \\
[4-1]P1(2.5)/[4-2]P1(1.5) & 0.11 & 0.063 & 0.059 \\
[4-1]R1(1.5)/[4-2]R1(1.5) & 0.12 & 0.063 & 0.061 \\
[5-2]P1(2.5)/[5-3]P1(2.5) & 0.17 & 0.096 & 0.097 \\
[5-2]R1(1.5)/[5-3]R1(1.5) & 0.17 & 0.098 & 0.10 \\
[5-2]Q2(0.5)/[5-3]Q2(0.5) & 0.18 & 0.097 & 0.099 \\
[9-5]Q1(1.5)/[9-7]Q1(1.5) & 0.21 & 0.058 & 0.075 \\
[9-5]P1(2.5)/[9-7]P1(2.5) & 0.23 & 0.056 & 0.070 \\
& & & \\
[4-1]R1(2.5)/[4-1]P1(4.5) & 0.55 & 0.54 & 0.61 \\
[4-2]P1(2.5)/[4-2]Q1(1.5) & 0.76 & 0.76 & 0.74 \\
[5-2]Q2(0.5)/[5-2]P2(1.5) & 0.50 & 0.49 & 0.50 \\
[5-3]R1(2.5)/[5-3]P1(4.5) & 0.55 & 0.53 & 0.57 \\
[6-4]Q1(2.5)/[6-4]R1(1.5) & 0.81 & 0.98 & 0.96 \\
[7-4]R1(2.5)/[7-4]P1(4.5) & 0.55 & 0.56 & 0.61 \\
[8-5]P1(2.5)/[8-5]Q1(1.5) & 0.73 & 0.74 & 0.72 \\
[9-5]R1(1.5)/[9-5]P1(3.5) & 0.41 & 0.46 & 0.51 \\
[9-7]R2(1.5)/[9-7]P2(3.5) & 0.49 & 0.50 & 0.51 \\
\hline
\end{tabular}

Notes. ${ }^{(1)}$ First group are pairs of lines with different $\Delta v$, second group are pairs of lines with the same $\Delta v{ }^{(2)}$ First entry is based on the transition probabilities of van de Loo (2007), second entry is taken from the R2000 list, which is based on the transition probabilities of Mies (1974).

strong contribution of $\mathrm{O}_{2}$ lines in the $J$-band (see Sect. 3.4). The $Y-H$ colour, instead, can be accurately modelled because $\mathrm{OH}$ accounts for most of the emission in both bands. The airglow in the $Y$-band is mostly due to $\mathrm{OH}$ lines with $\Delta v=3$ (3-0 and 4-1), while the $H$-band only contains $\mathrm{OH}$ lines with $\Delta v=2$ (bands from 2-0 to 6-4). Therefore, apart from minor effects related to weak temporal variations of the vibrational and rotational temperatures, the $Y-H$ colour should have a quasiconstant value that solely depends on the relative transition probabilities between these vibrational bands. Using the published transition probabilities, we expect a photon-flux ratio $N_{\lambda}(H) / N_{\lambda}(Y)=10.8$, equivalent to a colour $Y-H=3.8$. The data in Fig. 5 confirm the predicted stability of the $Y-H$ colour but, most important, shows that the airglow emission is $0.8 \mathrm{mag}$ bluer than expected. In other words, the lines in the $Y$ band are, on average, a factor of 2 brighter than predicted. This is the same result as the one we found in the GIANO spectrum.

\section{4. $\mathrm{O}_{2}$ and unidentified lines}

The GIANO spectrum includes about 150 lines that cannot be associated with $\mathrm{OH}$ transitions. The measured line positions and fluxes are summarised in Table 3. The table lists the observed wavelengths (in vacuum), which are accurate to $0.05 \AA$ rms. The relative photon fluxes and the accuracy flags ( $a=$ best, $c=$ worst) are in the same unit as the $\mathrm{OH}$ lines (Sect. 3.1).

All brightest lines in the $J$-band are identified as rotovibrational transitions of the $\mathrm{O}_{2}(0,0) a^{1} \Delta_{g}-X^{3} \Sigma_{g}^{-}$band. The observed wavelengths are equal within the errors to those listed in the HITRAN database (Rothman et al. 2009). Most of these lines are coincident with telluric absorption features, i.e. the $\mathrm{O}_{2}$ lines are optically thick.

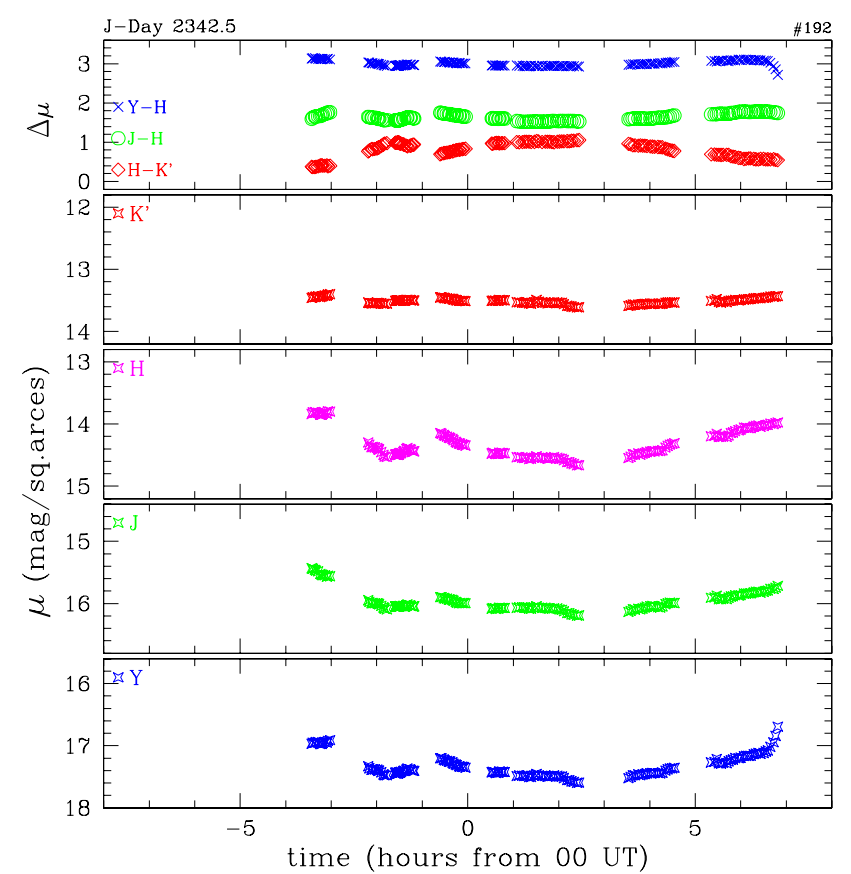

Fig. 5. Variation of the airglow emission at La Palma during a dark night (moon 22\%, rising at 04:45 UT). The values of surface brightness and colours are expressed in Vega-magnitudes. The $Y-H$ colours (uppermost points) are much bluer than predicted by theoretical $\mathrm{OH}$ spectra. See text for details.

The brightest emission features at longer wavelengths are the broad features at 1.5803 and $1.5808 \mu \mathrm{m}$ (see top panel of Fig. 6). These features are the band-heads of the $(0,1) a^{1} \Delta_{g}-X^{3} \Sigma_{g}^{-}$ transitions of $\mathrm{O}_{2}$. This is the first overtone of the $(0,0)$ band discussed above. Most of the lines in the 1.56-1.61 $\mu \mathrm{m}$ range are also coincident with $\mathrm{O}_{2}$ transitions listed in the HITRAN database. Interestingly, none of these emission lines is coincident with telluric absorption features. Therefore, unlike the $(0,0)$ $\mathrm{O}_{2}$ band, it seems that the $(0,1) \mathrm{O}_{2}$ lines are optically thin.

The remaining lines are unlikely to be associated with the $\mathrm{O}_{2}$ band, because they are very far from the $\mathrm{O}_{2}$ band-heads. An intriguing result is that several of these lines appear as closely spaced doublets with equal intensities (see Fig. 6). In other words, they are very similar to the $\Lambda$-split $\mathrm{OH}$ doublets detected in our spectra. However, their wavelengths do not correspond to any $\mathrm{OH}$ transition with $J_{\mathrm{u}} \leq 19.5$ and $v_{\mathrm{u}} \leq 10$. The possibility that these doublets could be produced by $\mathrm{OH}$ isotopologues (e.g. ${ }^{18} \mathrm{OH}$ ) should be investigated, but is beyond the aims of this paper.

It is interesting to note that the integrated photon flux due to non-OH lines is about $5 \%$ of the total airglow line emission in the $H$-band. Specifically, about $1.5 \%$ is accounted for by the two band-heads around $1.58 \mu \mathrm{m}$, while the remaining $3.5 \%$ is in isolated lines. This contribution is not necessarily negligible and could complicate the design of airglow-subtraction devices for astronomical instruments.

We did not include the broad emission features at wavelengths beyond $2.3 \mu \mathrm{m}$ in the list, which are produced by absorption bands generated at relatively low heights in the atmosphere.

\section{Conclusions}

Using GIANO at the TNG, we have obtained a high-resolution $(R \simeq 50000)$ flux-calibrated spectrum of the night airglow 


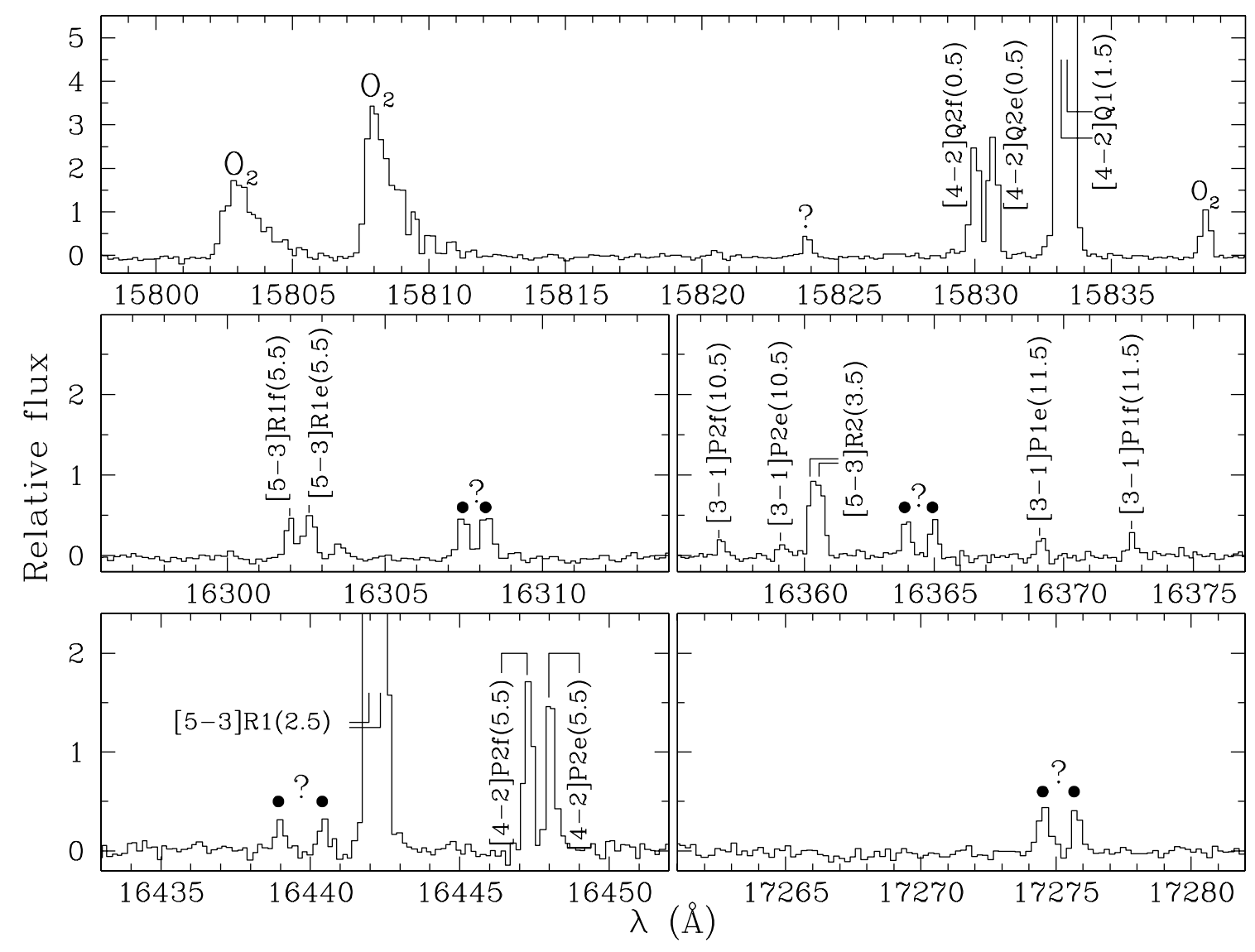

Fig. 6. Top panel: section of the GIANO spectrum including the band-heads of $\mathrm{O}_{2}(0,1) a^{1} \Delta_{g}-X^{3} \Sigma_{g}^{-}$. Lower panels: unidentified lines that appear as $\Lambda$-split doublets. See text for details.

covering the $0.95-2.4 \mu \mathrm{m}$ wavelength range. To the best of our knowledge, this is the first spectrum of this type ever taken.

About $80 \%$ of the detected lines can be unambiguously identified as $\mathrm{OH}$ transitions. The observed wavelengths agree with those expected by the most accurate molecular energy levels available in the literature (Abrams et al. 1994).

The relative fluxes of $\mathrm{OH}$ are used to determine the physical conditions of the emitting molecules. Most of the data are well fitted by a standard model, where the population of the vibrational states follows a Boltzmann distribution at $T_{\text {vib }}=9000 \mathrm{~K}$, while the rotational levels within a given vibrational state are thermalised at $T_{\text {rot }}=180 \mathrm{~K}$. However, we also detected lines from highly excited rotational levels. These reveal a population of hot $\mathrm{OH}$, with $T_{\text {rot }} \simeq T_{\text {vib }}$, which accounts for a few $\%$ of the total number of molecules. This result indicates that the timescales for the thermalisation of the rotational levels are not short enough to completely quench the emission from recently formed molecules in highly excited rotational states.

Most surprisingly, the relative intensities of $\mathrm{OH}$ lines from the same upper level show considerable discrepancies with that predicted by computed transition probabilities.

All non-OH lines observed in the $1.2-1.3 \mu \mathrm{m}$ range can be identified as $\mathrm{O}_{2}$ transitions within the $(0,0) a^{1} \Delta_{g}-$ $X^{3} \Sigma_{g}^{-}$band. The remaining non-OH airglow lines are in the $H$-band $(1.5-1.8 \mu \mathrm{m})$. Of these, about $2 / 3$ are associated with the first overtone of the same $\mathrm{O}_{2}$ band, i.e. $(0,1) a^{1} \Delta_{g}-X^{3} \Sigma_{q}^{-}$ at $1.58 \mu \mathrm{m}$. Interestingly, these lines are not coincident with telluric absorption features, i.e. the lines are, most probably, optically thin. The remaining lines, being far from the $\mathrm{O}_{2}$ bandheads, are unlikely to be associated with these band.
Acknowledgements. Part of this work was supported by the grant TECNOINAF-2011.

\section{References}

Abrams, M. C., Davis, S. P., Rao, M. L. P., Engleman, R. Jr., \& Brault, J. W. 1994, ApJS, 93, 351

Baffa, C., Comoretto, G., Gennari, S., et al. 2001, A\&A, 378, 722

Cosby, P. C., Sharpee B. D., Slanger, T. G., Huestis, D. L., \& Hanuschik, R. W. 2006, J. Geophys. Res., 111, 2307

Ellis, S. C., Bland-Hawthorn, J., Lawrence, J., et al. 2012, MNRAS, 425, 1682

Hanuschik, R. W. 2003, A\&A, 407, 1157

Kaye, J. A. 1988, J. Geophys. Res., 93, 285

Kramida, A., Ralchenko, Yu., Reader, J., \& NIST ASD Team 2012, NIST Atomic Spectra Database (version 5.0), National Institute of Standards and Technology, Gaithersburg, MD, available: http://physics.nist.gov/ asd

Makhlouf, U. B., Picard, R. H., \& Winick, R. J. 1995, J. Geophys. Res., 100, 11289

Maihara, T., Iwamuro, F., Yamashita, T., et al. 1993, PASP, 105, 940

Mies, F. H. 1974, J. Mol. Spec., 53, 150

Oliva, E., \& Origlia, L. 1992, A\&A, 254, 466

Oliva, E., Origlia, L., Maiolino, R., et al. 2012a, SPIE, 8446, 3

Oliva, E., Biliotti, V., Baffa, C., et al. 2012b, SPIE, 8453, 2

Osterbrock, D. E., Donald, E., Fulbright, J. P., Cosby, P. C., \& Barlow, T. A. 1998, PASP, 110, 1499

Redman, S. L., Lawler, J. E., Nave, G., Ramsey, L. W., \& Mahadevan, S. 2011, ApJS, 195, 24

Rothman, L. S., Gordon, I. E., Barbe, A., et al. 2009, JQS\&RT, 110, 533

Rousselot, P., Lidman, C., Cuby, J.-G., Moreels, G., \& Monnet, G. 2000, A\&A, 354, 1134 (R2000)

Sharma, R. D. 1985, Handbook of Geophysics, Chap. 13 (Air Force Geophysics Laboratory, USAF)

van der Loo, M. P. J., \& Groenenboom, G. C. 2007, J. Chem. Phys., 126, 4314 